\title{
Supporting Information \\ Role of Explicit Solvation in the Simulation of Resonance Raman Spectra within Short-time Dynamics Approximation
}

\author{
Sayan Mondal a,b*, and Chandrabhas Narayana a,c* \\ Jawaharlal Nehru Centre for Advanced Scientific Research, \\ Bangalore - 560064, Karnataka, India
}

aJawaharlal Nehru Centre for Advanced Scientific Research, Bangalore - 560064, India

${ }^{b}$ Current Address: Department of Chemical and Biological Physics, Weizmann Institute of Science, Rehovot 76100, Israel

'School of Advanced Materials, Jawaharlal Nehru Centre for Advanced Scientific Research, Bangalore - 560064, Karnataka, India

*Corresponding Author: Sayan Mondal, Chandrabhas Narayana

Email: sayanmondal.ncbs@gmail.com; cbhas@jncasr.ac.in

Reprint Author: Sayan Mondal, sayanmondal.ncbs@gmail.com 


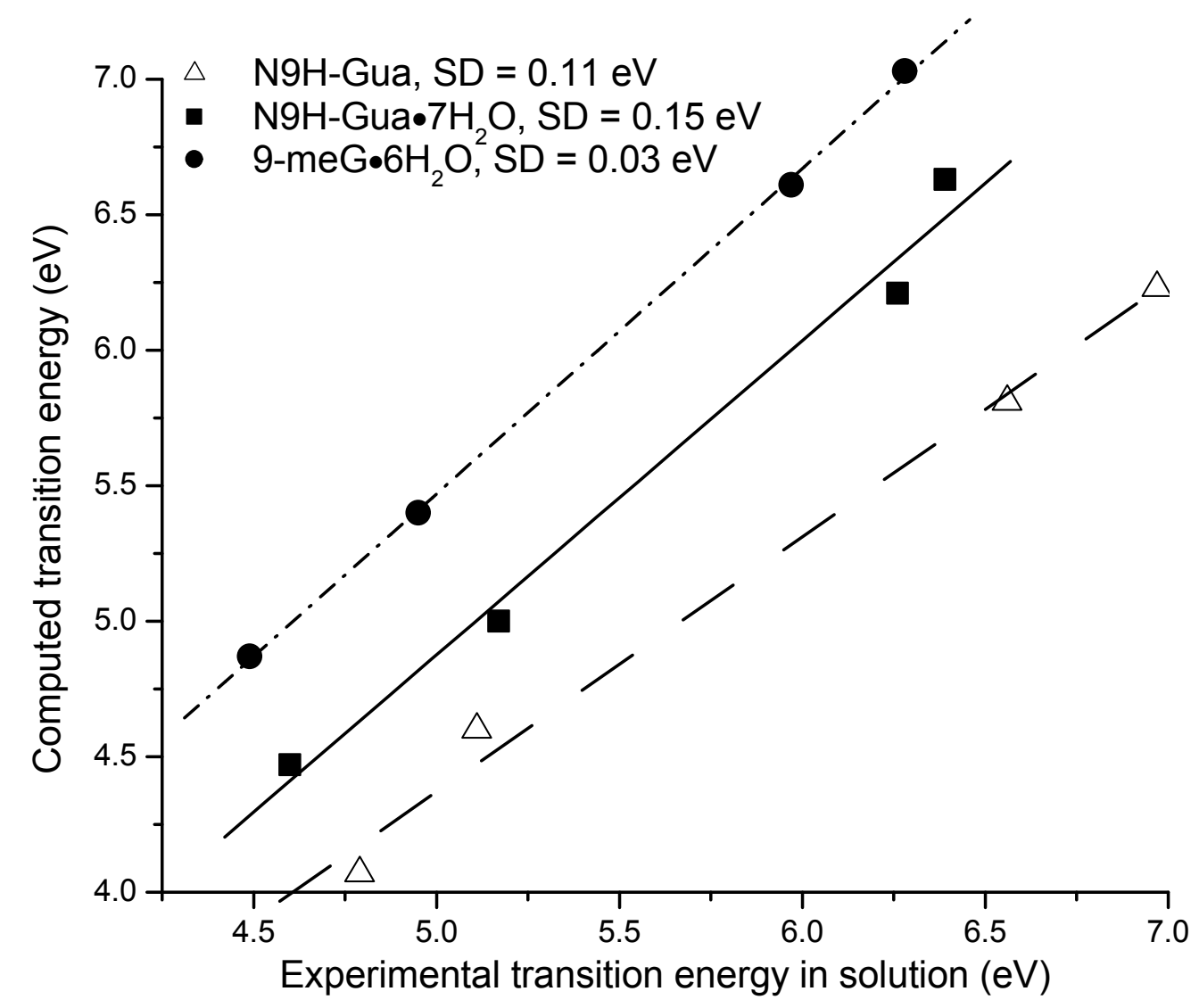

Figure S1. Linear regression fit of computed vertical transition energies of four $\pi \pi^{*}$ states and average experimental transition energies of Gua chromophore. Excitation energies are computed using cluster-continuum model on $\mathrm{N}_{9} \mathrm{H}-\mathrm{Gua}, \mathrm{N}_{9} \mathrm{H}-\mathrm{Gua} \bullet 7 \mathrm{H}_{2} \mathrm{O}$ and $9-\mathrm{meGua} \bullet 6 \mathrm{H}_{2} \mathrm{O}$ models at TDB3LYP/6-311+G(2d,p)//PCM level of theory at ground state geometry optimized with B3LYP functional. Computed and average experimental transition energies are described in table 1. Data for different models have been offset along the ordinate for the sake of visual clarity. The depicted ordinate values correspond to the $\mathrm{N}_{9} \mathrm{H}-\mathrm{Gua} .7 \mathrm{H}_{2} \mathrm{O}$ model. 


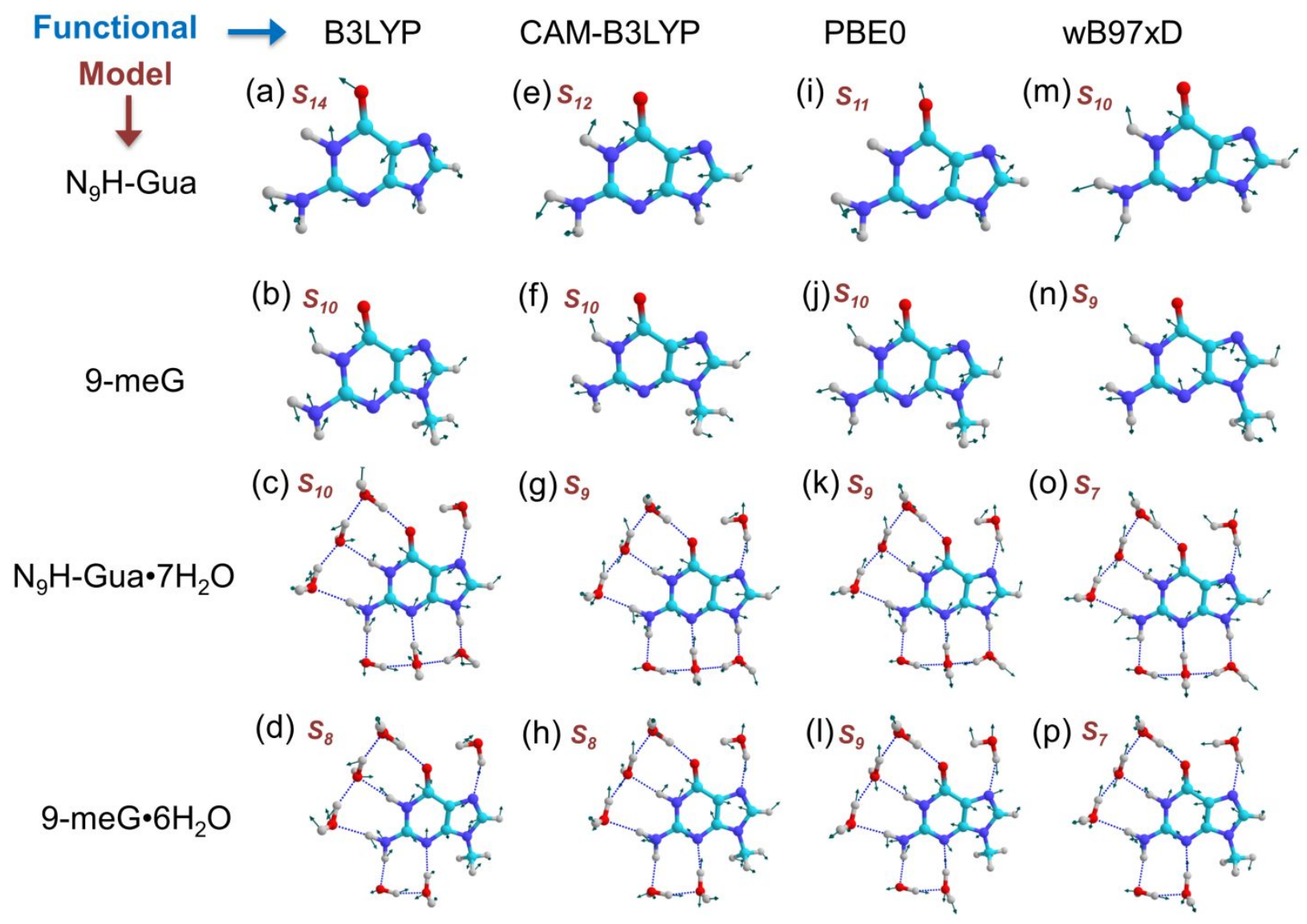

Figure S2. Direction of cartesian forces on all atoms of Gua in FC region of $B_{b}$ electronic state, computed on four models with (a-d) B3LYP; (e-h) CAM-B3LYP; (i-1) PBE0 and (m-p) wB97xD functionals. Ground state structure was optimized with B3LYP functional. Both ground state normal modes and excited state gradients are computed with $6-311+\mathrm{G}(2 \mathrm{~d}, \mathrm{p})$ basis set and PCM implicit solvation. Calculated state order of $B_{b}$ excited state in all models are labelled in bold crimson red italic font. 
(e)

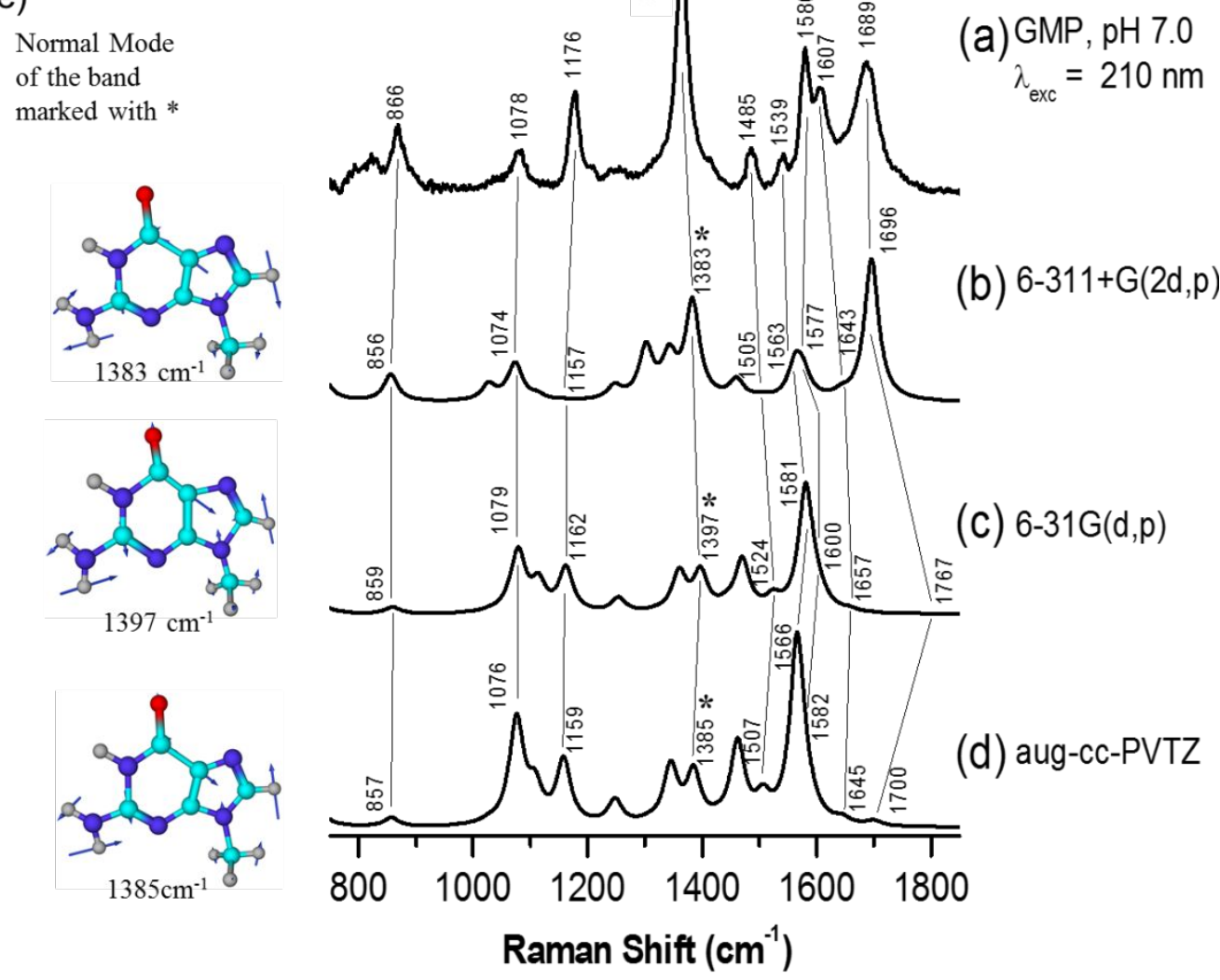

Figure S3. Dependence of predicted RR spectrum of isolated 9-meG on choice of basis set. All spectra (on isolated 9-meG in $B_{b}$ electronic state) were obtained using ground state normal modes computed with B3LYP functional in conjunction with (b) 6-311+G(2d,p), (c) 6-31G(d,p) and (d) aug-cc-PVTZ basis set, (e) computed ground state normal mode corresponding to 1365 $\mathrm{cm}^{-1}$ experimental band with three aforementioned basis sets. For all three computed spectra excited state gradient FC region of $B_{b}$ excited state (computed as $S_{10}$ in isolated 9-meG) is evaluated at TD-B3LYP/6-311+G(2d,p)//PCM level at ground state equilibrium geometry. The experimental spectrum of GMP (1 mM, miliQ Water, $\mathrm{pH} 6.8)$ at $210 \mathrm{~nm}$ excitation wavelength in shown in top (a) for comparison. Lorentzian line-shape with FWHM of $15 \mathrm{~cm}^{-1}$ is used for generating spectra form computed intensities. No frequency scaling was used. 


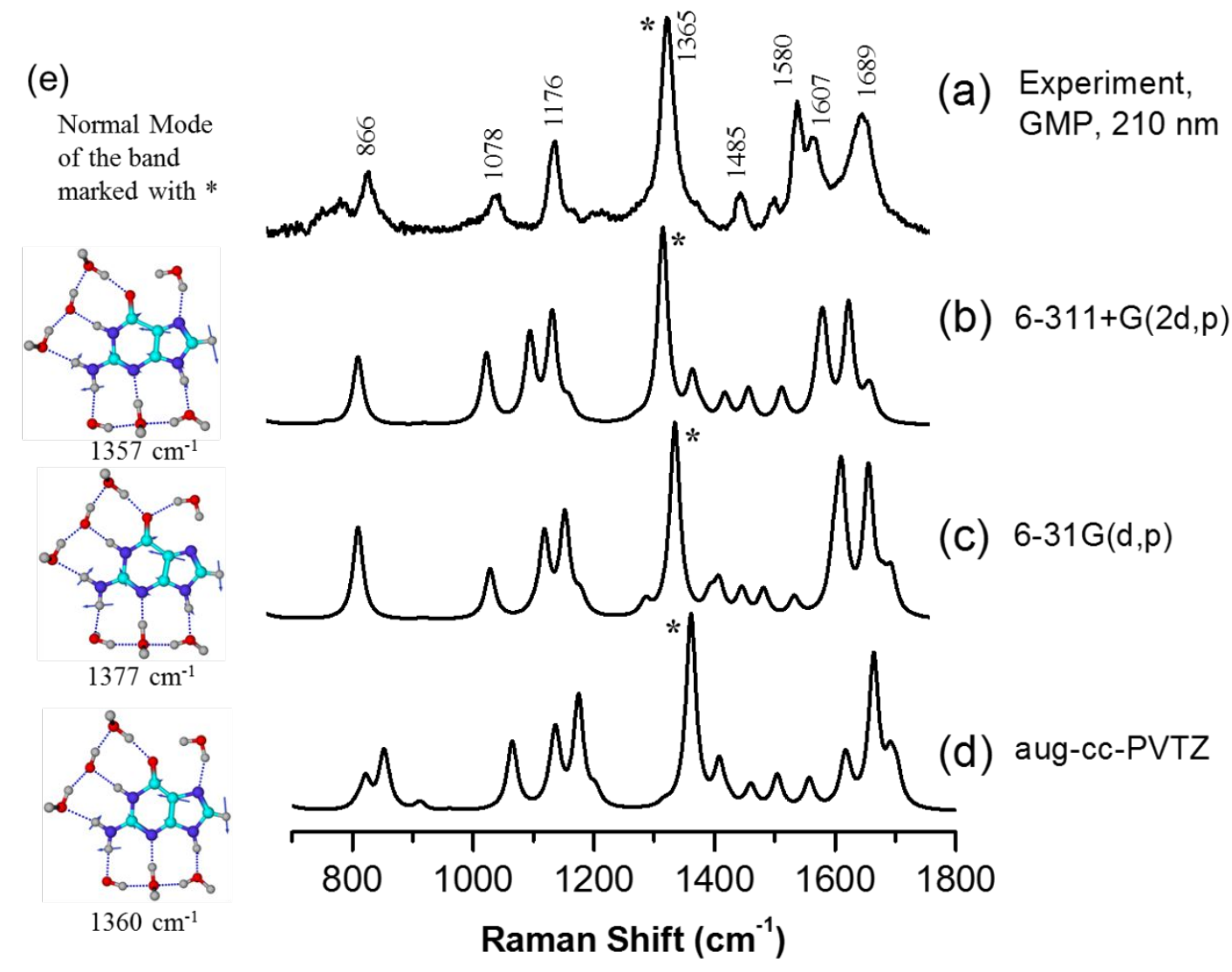

Figure S4. Dependence of predicted RR spectrum of $\mathrm{N}_{9} \mathrm{H}-\mathrm{Gua} \cdot 7 \mathrm{H}_{2} \mathrm{O}$ on choice of basis set. All spectra (on $\mathrm{N}_{9} \mathrm{H}-\mathrm{Gua} \cdot 7 \mathrm{H}_{2} \mathrm{O}$ model in $B_{b}$ electronic state) were obtained using ground state normal modes computed with B3LYP functional in conjunction with (b) 6-311+G(2d,p), (c) 6$31 \mathrm{G}(\mathrm{d}, \mathrm{p})$ and $(\mathrm{d})$ aug-cc-PVTZ basis set, (e) computed ground state normal mode corresponding to $1365 \mathrm{~cm}^{-1}$ band with three aforementioned basis sets. For all three computed spectra excited state gradient on $B_{b}$ excited state $\left(S_{10}\right.$ in isolated 9-meG) is evaluated at TD-B3LYP/6$311+\mathrm{G}(2 \mathrm{~d}, \mathrm{p}) / / \mathrm{PCM}$ level at ground state equilibrium geometry. The experimental spectrum of GMP at $210 \mathrm{~nm}$ excitation wavelength in shown in top (a) for comparison. Lorentzian line-shape with FWHM of $15 \mathrm{~cm}^{-1}$ is used for generating spectra form computed intensities. No frequency scaling was used. Refer to Table S12 for excitation energies, oscillator strengths, and orbitals contribution for three $\pi-\pi^{*}$ electronic states of GMP, computed of $\mathrm{N}_{9} \mathrm{H}-\mathrm{Gua} \cdot 7 \mathrm{H}_{2} \mathrm{O}$ model on three different ground state geometry with three basis set. 

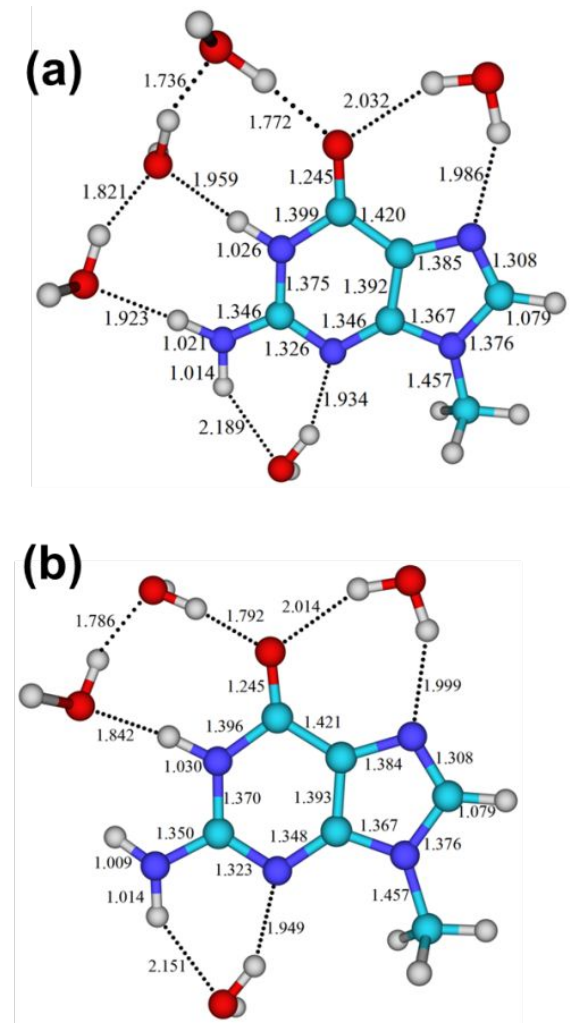

Figure S5. Ground state equilibrium structure of (a) $9-\mathrm{meG} \cdot 5 \mathrm{H}_{2} \mathrm{O}$ and (b) $9-\mathrm{meG} \cdot 4 \mathrm{H}_{2} \mathrm{O}$ model complex after energy minimization with B3LYP functional and $6-311+\mathrm{G}(2 \mathrm{~d}, \mathrm{p})$ Gaussian basis set with PCM implicit solvation. 


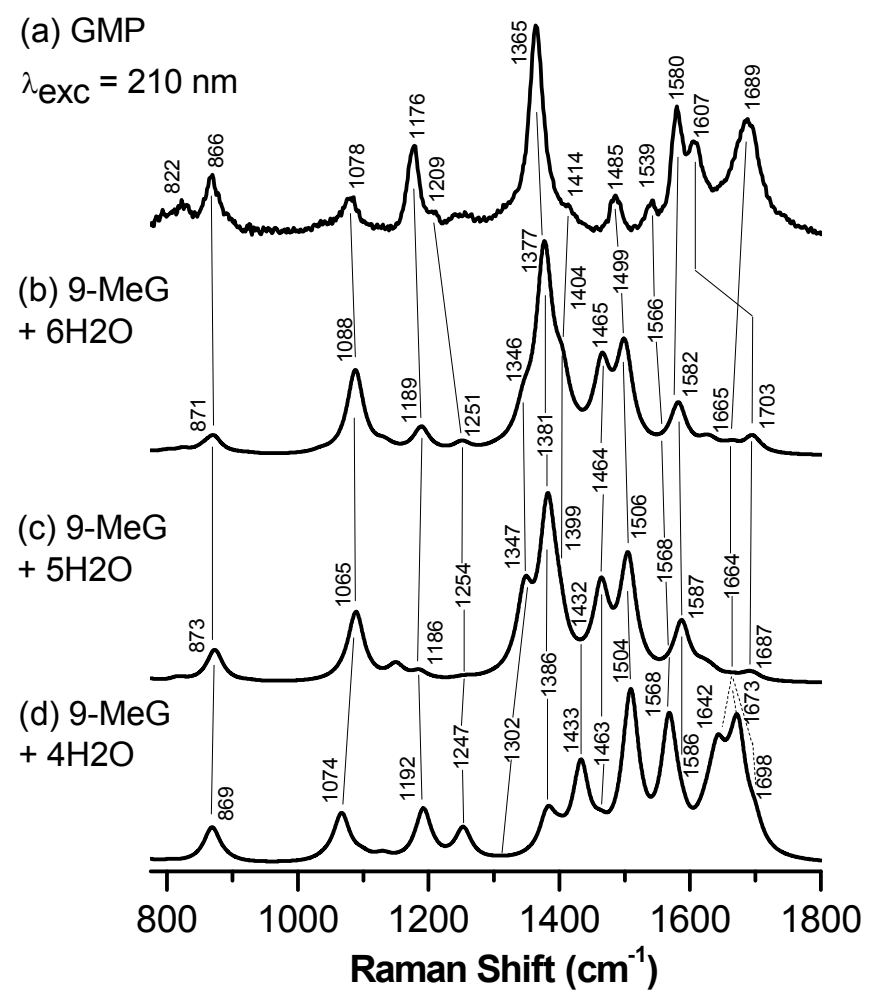

Figure S6. Computed RR spectra of (b) $9-m e G \bullet 6 \mathrm{H}_{2} \mathrm{O}$, (c) $9-\mathrm{meG} \cdot 5 \mathrm{H}_{2} \mathrm{O}$ and (d) $9-\mathrm{meG} \cdot 4 \mathrm{H}_{2} \mathrm{O}$ model complex in $B_{b}$ electronic state with TD-CAM-B3LYP derived excited state gradients. The experimental spectrum at top (a) in both panels is of $1 \mathrm{mM} \mathrm{GMP}$ (in water, pH 6.8) with 210 $\mathrm{nm}$ excitation wavelength. For calculation of these spectra ground state normal modes of all four models are computed with B3LYP functional. For evaluation of excited state gradients same basis set was used. All ground and excited state calculations are performed with $6-311+\mathrm{G}(2 \mathrm{~d}, \mathrm{p})$ basis set and PCM as implicit solvation model. Lorentzian line-shape with FWHM of $15 \mathrm{~cm}^{-1}$ is used for generating spectra form computed intensities. No frequency scaling was used. 

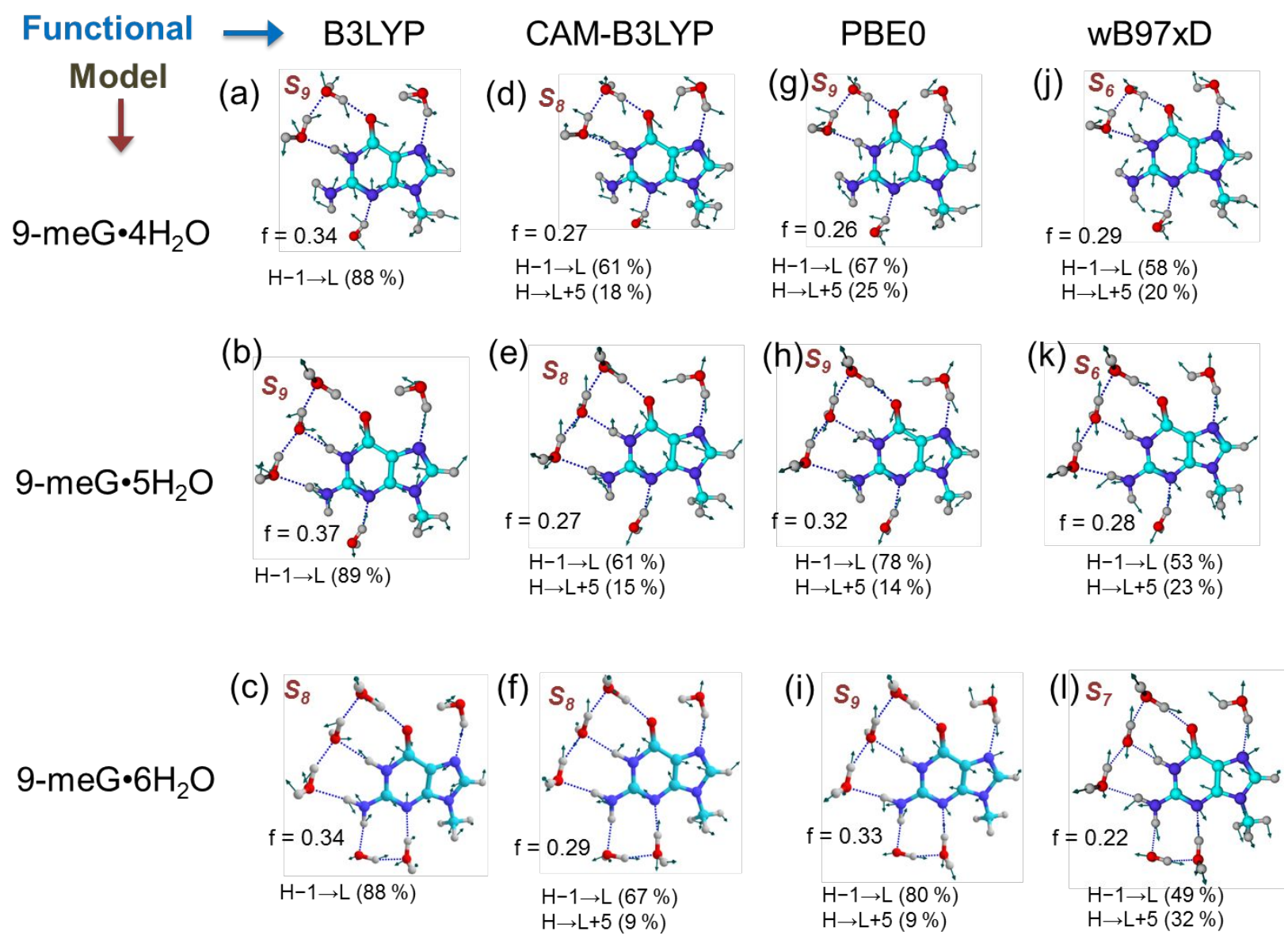

Figure S7. Cartesian forces on atoms of GMP in FC region of $B_{b}$ electronic state, computed on three models with (a-c) B3LYP; (d-f) CAM-B3LYP; (g-i) PBE0 and (j-1) wB97xD functionals. Ground state structure was optimized with B3LYP functional. Both ground state normal modes and excited state gradients are computed with $6-311+\mathrm{G}(2 \mathrm{~d}, \mathrm{p})$ basis set and PCM implicit solvation. Calculated state order of $B_{b}$ excited state in all models are labelled in bold crimson red italic font. Oscillator strengths (f) and orbital contribution in each transitions are also mentioned in each case, $\mathrm{H}$ and $\mathrm{L}$ stand for highest occupied molecular orbital (HOMO) and lowest unoccupied molecular orbital (LUMO) respectively. 
Table S1. Cartesian coordinates of ground state equilibrium geometry of neutral $\mathrm{N}_{9} \mathrm{H}-\mathrm{Gua}$ computed with B3LYP functional and 6-311+G(2d,p) Gaussian basis set with PCM implicit solvation.

\begin{tabular}{|l|c|c|c|}
\hline $\mathrm{N}$ & -1.47648 & 0.78998 & 0.00020 \\
\hline $\mathrm{C}$ & -1.67500 & -0.56716 & -0.00120 \\
\hline $\mathrm{N}$ & -0.68774 & -1.43484 & 0.00486 \\
\hline $\mathrm{C}$ & 0.53108 & -0.85466 & 0.00035 \\
\hline $\mathrm{C}$ & 0.84256 & 0.50171 & 0.00070 \\
\hline $\mathrm{C}$ & -0.22485 & 1.44822 & 0.00101 \\
\hline $\mathrm{N}$ & 1.73753 & -1.49540 & 0.00103 \\
\hline $\mathrm{C}$ & 2.71050 & -0.52218 & 0.00071 \\
\hline $\mathrm{N}$ & 2.21411 & 0.68755 & 0.00077 \\
\hline $\mathrm{N}$ & -2.96104 & -1.00503 & -0.06192 \\
\hline $\mathrm{H}$ & 3.75933 & -0.77402 & 0.00063 \\
\hline $\mathrm{H}$ & 1.88552 & -2.49416 & 0.00085 \\
\hline $\mathrm{H}$ & -3.70318 & -0.39737 & 0.25077 \\
\hline $\mathrm{H}$ & -3.09427 & -1.98500 & 0.13856 \\
\hline $\mathrm{O}$ & -0.18232 & 2.67654 & 0.00062 \\
\hline $\mathrm{H}$ & -2.27922 & 1.40685 & -0.01972 \\
\hline
\end{tabular}


Table S2. Cartesian coordinates of ground state equilibrium geometry of neutral isolated 9-meG computed with B3LYP functional and $6-311+\mathrm{G}(2 \mathrm{~d}, \mathrm{p})$ Gaussian basis set with PCM implicit solvation.

\begin{tabular}{|r|r|r|r|}
\hline $\mathrm{O}$ & 1.637992000 & 2.506103000 & 0.000217000 \\
\hline $\mathrm{N}$ & -1.928097000 & -0.405549000 & 0.000687000 \\
\hline $\mathrm{N}$ & 0.277848000 & -1.406670000 & 0.004656000 \\
\hline $\mathrm{N}$ & -1.390411000 & 1.774610000 & 0.002669000 \\
\hline $\mathrm{N}$ & 1.966346000 & 0.241874000 & -0.002014000 \\
\hline $\mathrm{N}$ & 2.507657000 & -2.025837000 & -0.061088000 \\
\hline $\mathrm{C}$ & -0.561824000 & -0.348414000 & -0.000019000 \\
\hline $\mathrm{C}$ & -2.365113000 & 0.900648000 & 0.001802000 \\
\hline $\mathrm{C}$ & -0.241937000 & 1.006593000 & 0.001395000 \\
\hline $\mathrm{C}$ & 1.132934000 & 1.384870000 & 0.000649000 \\
\hline $\mathrm{C}$ & 1.546182000 & -1.063958000 & -0.001616000 \\
\hline $\mathrm{H}$ & -3.418941000 & 1.134255000 & 0.002257000 \\
\hline $\mathrm{H}$ & 3.438795000 & -1.811014000 & 0.263067000 \\
\hline $\mathrm{H}$ & 2.190507000 & -2.962289000 & 0.141368000 \\
\hline $\mathrm{H}$ & 2.958374000 & 0.442970000 & -0.025264000 \\
\hline $\mathrm{C}$ & -2.748020000 & -1.609098000 & -0.001065000 \\
\hline $\mathrm{H}$ & -2.544427000 & -2.202464000 & -0.891876000 \\
\hline $\mathrm{H}$ & -2.539982000 & -2.207912000 & 0.885029000 \\
\hline $\mathrm{H}$ & -3.794995000 & -1.315211000 & 0.002436000 \\
\hline
\end{tabular}


Table S3. Cartesian coordinates of ground state equilibrium geometry of neutral $\mathbf{N}_{9} \mathbf{H}-\mathbf{G u a} \cdot 7 \mathrm{H}_{2} \mathrm{O}$ model computed with B3LYP functional and 6-311+G(2d,p) Gaussian basis set with PCM implicit solvation.

\begin{tabular}{|c|c|c|c|}
\hline $\mathrm{N}$ & 1.018502000 & 0.486235000 & -0.121658000 \\
\hline $\mathrm{C}$ & -0.241407000 & 1.042089000 & -0.114483000 \\
\hline $\mathrm{N}$ & -1.340326000 & 0.295875000 & -0.070173000 \\
\hline $\mathrm{C}$ & -1.104629000 & -1.033283000 & -0.058872000 \\
\hline $\mathrm{C}$ & 0.130354000 & -1.674633000 & -0.036948000 \\
\hline $\mathrm{C}$ & 1.306994000 & -0.881128000 & -0.059593000 \\
\hline $\mathrm{N}$ & -2.036111000 & -2.030148000 & -0.045150000 \\
\hline $\mathrm{C}$ & -1.344272000 & -3.215468000 & -0.016513000 \\
\hline $\mathrm{N}$ & -0.046035000 & -3.049314000 & -0.008324000 \\
\hline $\mathrm{N}$ & -0.328504000 & 2.376856000 & -0.173427000 \\
\hline $\mathrm{H}$ & -1.852359000 & -4.166778000 & -0.003199000 \\
\hline $\mathrm{H}$ & -3.058115000 & -1.911246000 & -0.072002000 \\
\hline $\mathrm{H}$ & 0.471694000 & 2.978028000 & 0.024465000 \\
\hline $\mathrm{H}$ & -1.250947000 & 2.809084000 & -0.200458000 \\
\hline $\mathrm{O}$ & 2.489943000 & -1.268765000 & -0.042798000 \\
\hline $\mathrm{H}$ & 1.824005000 & 1.104868000 & -0.260205000 \\
\hline $\mathrm{O}$ & 4.826061000 & 0.098684000 & 0.417152000 \\
\hline $\mathrm{H}$ & 5.051318000 & 0.108144000 & 1.355025000 \\
\hline $\mathrm{H}$ & 4.014275000 & -0.451385000 & 0.334184000 \\
\hline $\mathrm{O}$ & 3.442476000 & 2.173847000 & -0.612293000 \\
\hline $\mathrm{H}$ & 4.088509000 & 1.535020000 & -0.222841000 \\
\hline $\mathrm{H}$ & 3.698260000 & 2.293462000 & -1.535023000 \\
\hline $\mathrm{O}$ & 1.905527000 & 4.207362000 & 0.419084000 \\
\hline $\mathrm{H}$ & 2.629707000 & 3.601340000 & 0.162344000 \\
\hline $\mathrm{H}$ & 2.055202000 & 4.420367000 & 1.347624000 \\
\hline $\mathrm{O}$ & -3.008253000 & 3.698064000 & -0.282213000 \\
\hline $\mathrm{H}$ & -3.311836000 & 3.966240000 & -1.157515000 \\
\hline $\mathrm{H}$ & -3.538966000 & 2.911250000 & -0.046286000 \\
\hline $\mathrm{O}$ & -3.900135000 & 1.178362000 & 0.581495000 \\
\hline $\mathrm{H}$ & -2.965900000 & 0.886182000 & 0.394804000 \\
\hline $\mathrm{H}$ & -3.968409000 & 1.250389000 & 1.542632000 \\
\hline $\mathrm{O}$ & 2.605868000 & -4.195612000 & 0.004098000 \\
\hline $\mathrm{H}$ & 2.883719000 & -3.264887000 & -0.016079000 \\
\hline $\mathrm{H}$ & 1.633992000 & -4.089606000 & 0.006843000 \\
\hline $\mathrm{O}$ & -4.813721000 & -1.362474000 & -0.179314000 \\
\hline $\mathrm{H}$ & -5.435099000 & -1.764860000 & 0.439317000 \\
\hline $\mathrm{H}$ & -4.706092000 & -0.433346000 & 0.114256000 \\
\hline & & & \\
\hline
\end{tabular}


Table S4. Cartesian coordinates of ground state equilibrium geometry of neutral $\mathbf{9}-\mathbf{m e G} \cdot \mathbf{6} \mathbf{H}_{\mathbf{2}} \mathbf{O}$ model computed with B3LYP functional and $6-311+\mathrm{G}(2 \mathrm{~d}, \mathrm{p})$ Gaussian basis set with PCM implicit solvation.

\begin{tabular}{|c|c|c|c|}
\hline N & 0.712208000 & 0.682435000 & -0.156262000 \\
\hline $\mathrm{C}$ & -0.652624000 & 0.870915000 & -0.183152000 \\
\hline $\mathrm{N}$ & -1.508828000 & -0.146214000 & -0.142580000 \\
\hline $\mathrm{C}$ & -0.914955000 & -1.356807000 & -0.098489000 \\
\hline $\mathrm{C}$ & 0.448117000 & -1.634668000 & -0.038584000 \\
\hline $\mathrm{C}$ & 1.362830000 & -0.550110000 & -0.058176000 \\
\hline $\mathrm{N}$ & -1.539956000 & -2.574570000 & -0.089572000 \\
\hline $\mathrm{C}$ & -0.541733000 & -3.519601000 & -0.022066000 \\
\hline $\mathrm{N}$ & 0.658106000 & -3.001802000 & 0.011646000 \\
\hline $\mathrm{N}$ & -1.091206000 & 2.133087000 & -0.273376000 \\
\hline $\mathrm{H}$ & -0.771703000 & -4.573678000 & -0.003856000 \\
\hline $\mathrm{H}$ & -0.481208000 & 2.925839000 & -0.072843000 \\
\hline $\mathrm{H}$ & -2.093316000 & 2.317068000 & -0.287011000 \\
\hline $\mathrm{O}$ & 2.606274000 & -0.599093000 & -0.011765000 \\
\hline $\mathrm{H}$ & 1.320048000 & 1.497412000 & -0.287684000 \\
\hline $\mathrm{O}$ & 4.456370000 & 1.359278000 & 0.517534000 \\
\hline $\mathrm{H}$ & 4.626108000 & 1.440784000 & 1.463576000 \\
\hline $\mathrm{H}$ & 3.830931000 & 0.606654000 & 0.413763000 \\
\hline $\mathrm{O}$ & 2.601100000 & 2.960936000 & -0.611828000 \\
\hline $\mathrm{H}$ & 3.381015000 & 2.529009000 & -0.184899000 \\
\hline $\mathrm{H}$ & 2.850180000 & 3.131272000 & -1.528405000 \\
\hline $\mathrm{O}$ & 0.538425000 & 4.521891000 & 0.320418000 \\
\hline $\mathrm{H}$ & 1.409119000 & 4.133177000 & 0.100886000 \\
\hline $\mathrm{H}$ & 0.592211000 & 4.785818000 & 1.246368000 \\
\hline $\mathrm{O}$ & -3.961253000 & 2.912640000 & -0.194092000 \\
\hline $\mathrm{H}$ & -4.428580000 & 3.044203000 & -1.027183000 \\
\hline $\mathrm{H}$ & -4.244304000 & 2.031578000 & 0.136308000 \\
\hline $\mathrm{O}$ & -4.213443000 & 0.334353000 & 0.708300000 \\
\hline $\mathrm{H}$ & -3.289481000 & 0.110454000 & 0.447502000 \\
\hline $\mathrm{H}$ & -4.245594000 & 0.242550000 & 1.668143000 \\
\hline $\mathrm{O}$ & 3.529222000 & -3.372444000 & 0.055896000 \\
\hline $\mathrm{H}$ & 3.529097000 & -2.400955000 & 0.030847000 \\
\hline $\mathrm{H}$ & 2.568171000 & -3.548550000 & 0.052287000 \\
\hline $\mathrm{C}$ & -2.972597000 & -2.833823000 & -0.158579000 \\
\hline $\mathrm{H}$ & -3.401565000 & -2.322928000 & -1.019188000 \\
\hline $\mathrm{H}$ & -3.122951000 & -3.905210000 & -0.267436000 \\
\hline $\mathrm{H}$ & -3.468238000 & -2.490963000 & 0.748404000 \\
\hline & & & \\
\hline
\end{tabular}


Table S5. Cartesian coordinates of ground state equilibrium geometry of neutral $\mathbf{9}-\mathbf{m e G} \cdot \mathbf{5} \mathrm{H}_{2} \mathrm{O}$ model computed with B3LYP functional and $6-311+\mathrm{G}(2 \mathrm{~d}, \mathrm{p})$ Gaussian basis set with PCM implicit solvation.

\begin{tabular}{|c|c|c|c|}
\hline N & -0.903693000 & -0.209386000 & -0.159399000 \\
\hline $\mathrm{C}$ & -0.178664000 & -1.377394000 & -0.138115000 \\
\hline $\mathrm{N}$ & 1.145701000 & -1.391758000 & -0.066852000 \\
\hline $\mathrm{C}$ & 1.702520000 & -0.167152000 & -0.020332000 \\
\hline $\mathrm{C}$ & 1.051869000 & 1.063500000 & -0.015080000 \\
\hline $\mathrm{C}$ & -0.365918000 & 1.080287000 & -0.083725000 \\
\hline $\mathrm{N}$ & 3.037982000 & 0.117418000 & 0.049733000 \\
\hline $\mathrm{C}$ & 3.136715000 & 1.489388000 & 0.093526000 \\
\hline $\mathrm{N}$ & 1.976179000 & 2.091711000 & 0.057870000 \\
\hline $\mathrm{N}$ & -0.858177000 & -2.536330000 & -0.224058000 \\
\hline $\mathrm{H}$ & 4.096534000 & 1.979137000 & 0.150588000 \\
\hline $\mathrm{H}$ & -1.848187000 & -2.592747000 & 0.020143000 \\
\hline $\mathrm{H}$ & -0.304700000 & -3.378008000 & -0.111496000 \\
\hline $\mathrm{O}$ & -1.119294000 & 2.071426000 & -0.088461000 \\
\hline $\mathrm{H}$ & -1.918256000 & -0.264008000 & -0.299867000 \\
\hline $\mathrm{O}$ & -3.821966000 & 2.259037000 & 0.368719000 \\
\hline $\mathrm{H}$ & -4.010029000 & 2.401272000 & 1.304056000 \\
\hline $\mathrm{H}$ & -2.841797000 & 2.259270000 & 0.282030000 \\
\hline $\mathrm{O}$ & -3.853737000 & -0.256799000 & -0.604539000 \\
\hline $\mathrm{H}$ & -4.025715000 & 0.642111000 & -0.230596000 \\
\hline $\mathrm{H}$ & -4.157911000 & -0.234351000 & -1.520130000 \\
\hline $\mathrm{O}$ & -3.709959000 & -2.793487000 & 0.455982000 \\
\hline $\mathrm{H}$ & -3.972988000 & -1.887189000 & 0.197336000 \\
\hline $\mathrm{H}$ & -3.936593000 & -2.878816000 & 1.389460000 \\
\hline $\mathrm{O}$ & 1.712912000 & -4.185935000 & 0.148253000 \\
\hline $\mathrm{H}$ & 2.146167000 & -4.562456000 & -0.626592000 \\
\hline $\mathrm{H}$ & 1.785577000 & -3.213761000 & 0.044792000 \\
\hline $\mathrm{O}$ & 0.442183000 & 4.547298000 & -0.017630000 \\
\hline $\mathrm{H}$ & -0.311833000 & 3.935804000 & -0.056977000 \\
\hline $\mathrm{H}$ & 1.183459000 & 3.912070000 & 0.023928000 \\
\hline $\mathrm{C}$ & 4.134430000 & -0.841966000 & 0.077241000 \\
\hline $\mathrm{H}$ & 4.117708000 & -1.456982000 & -0.821720000 \\
\hline $\mathrm{H}$ & 5.072426000 & -0.293814000 & 0.118779000 \\
\hline $\mathrm{H}$ & 4.053375000 & -1.481414000 & 0.955527000 \\
\hline & & & \\
\hline
\end{tabular}


Table S6. Cartesian coordinates of ground state equilibrium geometry of neutral $\mathbf{9}-\mathbf{m e G} \cdot \mathbf{4} \mathbf{H}_{2} \mathrm{O}$ model computed with B3LYP functional and $6-311+\mathrm{G}(2 \mathrm{~d}, \mathrm{p})$ Gaussian basis set with PCM implicit solvation.

\begin{tabular}{|r|r|r|r|}
\hline $\mathrm{N}$ & 0.856673000 & -0.909018000 & -0.121170000 \\
\hline $\mathrm{C}$ & -0.346131000 & -1.563708000 & -0.099443000 \\
\hline $\mathrm{N}$ & -1.508692000 & -0.933810000 & -0.050766000 \\
\hline $\mathrm{C}$ & -1.389503000 & 0.408903000 & -0.032225000 \\
\hline $\mathrm{C}$ & -0.217057000 & 1.161006000 & -0.045246000 \\
\hline $\mathrm{C}$ & 1.027616000 & 0.475817000 & -0.083934000 \\
\hline $\mathrm{N}$ & -2.412445000 & 1.314219000 & 0.013323000 \\
\hline $\mathrm{C}$ & -1.824436000 & 2.558136000 & 0.026158000 \\
\hline $\mathrm{N}$ & -0.517374000 & 2.511478000 & -0.006871000 \\
\hline $\mathrm{N}$ & -0.305938000 & -2.911771000 & -0.163402000 \\
\hline $\mathrm{H}$ & -2.419394000 & 3.457719000 & 0.059657000 \\
\hline $\mathrm{H}$ & 0.563866000 & -3.390578000 & 0.015121000 \\
\hline $\mathrm{H}$ & -1.173043000 & -3.407447000 & 0.010356000 \\
\hline $\mathrm{O}$ & 2.174592000 & 0.960929000 & -0.086834000 \\
\hline $\mathrm{H}$ & 1.704502000 & -1.490984000 & -0.175683000 \\
\hline $\mathrm{O}$ & 4.528631000 & -0.435991000 & 0.332992000 \\
\hline $\mathrm{H}$ & 4.862452000 & -0.257178000 & 1.220098000 \\
\hline $\mathrm{H}$ & 3.730719000 & 0.128861000 & 0.224105000 \\
\hline $\mathrm{O}$ & 3.079276000 & -2.715632000 & -0.117852000 \\
\hline $\mathrm{H}$ & 3.756823000 & -2.025695000 & 0.070755000 \\
\hline $\mathrm{H}$ & 3.343873000 & -3.122226000 & -0.952049000 \\
\hline $\mathrm{O}$ & -3.305002000 & -3.154314000 & 0.147240000 \\
\hline $\mathrm{H}$ & -3.744546000 & -3.244596000 & 1.000819000 \\
\hline $\mathrm{H}$ & -2.938350000 & -2.246013000 & 0.133178000 \\
\hline $\mathrm{O}$ & 2.045431000 & 3.875417000 & -0.052644000 \\
\hline $\mathrm{H}$ & 2.382879000 & 2.963906000 & -0.071996000 \\
\hline $\mathrm{H}$ & 1.083559000 & 3.708236000 & -0.029808000 \\
\hline $\mathrm{C}$ & -3.838597000 & 1.016408000 & 0.044415000 \\
\hline $\mathrm{H}$ & -4.084375000 & 0.445963000 & 0.939442000 \\
\hline $\mathrm{H}$ & -4.387833000 & 1.954596000 & 0.055484000 \\
\hline $\mathrm{H}$ & -4.121465000 & 0.445105000 & -0.838850000 \\
\hline & & & \\
\hline
\end{tabular}


Table S7. Dihedral angle (a) $\mathrm{N}_{3} \mathrm{C}_{2} \mathrm{~N}_{2} \mathrm{H}_{2 b}$ and (b) $\mathrm{C}_{6} \mathrm{~N}_{1} \mathrm{C}_{2} \mathrm{~N}_{2}$ in four models of GMP. Decreasing value of these two dihedrals with increasing number of water molecules indicate that exocyclic $\mathrm{NH}_{2}$ moiety becomes more planar in presence of explicit solvent molecules through formation of hydrogen bonds. All computations are performed with B3LYP functional and 6-311+G(2d,p) Gaussian basis set with PCM implicit solvation. See Figure 1 in manuscript for atom numbering.

\begin{tabular}{|c|c|c|}
\hline GMP Model & $\mathbf{N}_{\mathbf{3}} \mathbf{C}_{\mathbf{2}} \mathbf{N}_{\mathbf{2}} \mathbf{H}_{\mathbf{2}}$ & $\mathbf{C}_{\mathbf{6}} \mathbf{N}_{\mathbf{1}} \mathbf{C}_{\mathbf{2}} \mathbf{N}_{\mathbf{2}}$ \\
\hline 9-meG & 12.7 & 2.68 \\
\hline 9-meG $\bullet 4 \mathrm{H}_{2} \mathrm{O}$ & 10.13 & 0.97 \\
\hline 9-meG・5 $\mathrm{H}_{2} \mathrm{O}$ & 5.94 & 0.16 \\
\hline 9-meG・6 $\mathrm{H}_{2} \mathrm{O}$ & 0.59 & 0.39 \\
\hline $\mathrm{N}_{9} \mathrm{H}-\mathrm{Gua} \cdot 7 \mathrm{H}_{2} \mathrm{O}$ & 1.99 & 0.35 \\
\hline
\end{tabular}


Table S8. Bond lengths (in $\AA$ ) of the purine ring in different models of GMP chromophore. All computations are performed with B3LYP functional and 6-311+G(2d,p) Gaussian basis set with PCM implicit solvation. See Figure 1a in manuscript for atom numbering.

\begin{tabular}{|c|c|c|c|c|c|c|c|}
\hline Bonds & $\begin{array}{c}\text { Crystal } \\
\text { Structure }^{1}\end{array}$ & $\mathrm{~N}_{9} \mathrm{H}-\mathrm{Gua}$ & 9-meG & $9-\mathrm{meG} \bullet 4 \mathrm{H}_{2} \mathrm{O}$ & $9-\mathrm{meG} \cdot 5 \mathrm{H} 2 \mathrm{O}$ & $9-\mathrm{meG} \bullet 6 \mathrm{H}_{2} \mathrm{O}$ & $\mathrm{N}_{9} \mathrm{H}-\mathrm{Gua} \cdot 7 \mathrm{H}_{2} \mathrm{O}$ \\
\hline $\mathrm{N}_{1}-\mathrm{C}_{2}$ & $\begin{array}{c}1.373 \pm \\
0.008\end{array}$ & 1.372 & 1.372 & 1.370 & 1.375 & 1.378 & 1.377 \\
\hline $\mathrm{C}_{2}-\mathrm{N}_{2}$ & $\begin{array}{c}1.341 \pm \\
0.010 \\
\end{array}$ & 1.360 & 1.361 & 1.350 & 1.346 & 1.339 & 1.339 \\
\hline $\mathrm{C}_{2}-\mathrm{N}_{3}$ & $\begin{array}{c}1.323 \pm \\
0.008 \\
\end{array}$ & 1.314 & 1.314 & 1.323 & 1.326 & 1.330 & 1.329 \\
\hline $\mathrm{N}_{3}-\mathrm{C}_{4}$ & $\begin{array}{c}1.350 \pm \\
0.007 \\
\end{array}$ & 1.350 & 1.351 & 1.348 & 1.346 & 1.349 & 1.350 \\
\hline $\mathrm{C}_{4}-\mathrm{C}_{5}$ & $\begin{array}{c}1.379 \pm \\
0.008\end{array}$ & 1.392 & 1.392 & 1.393 & 1.392 & 1.392 & 1.392 \\
\hline $\mathrm{C}_{5}-\mathrm{C}_{6}$ & $\begin{array}{c}1.419 \pm \\
0.010\end{array}$ & 1.427 & 1.426 & 1.421 & 1.420 & 1.419 & 1.419 \\
\hline $\mathrm{C}_{6}=\mathrm{O}_{6}$ & $\begin{array}{c}1.237 \pm \\
0.009\end{array}$ & 1.229 & 1.230 & 1.245 & 1.245 & 1.245 & 1.245 \\
\hline $\mathrm{C}_{6}-\mathrm{N}_{1}$ & $\begin{array}{c}1.391 \pm \\
0.007 \\
\end{array}$ & 1.414 & 1.415 & 1.396 & 1.399 & 1.397 & 1.399 \\
\hline $\mathrm{C}_{5}-\mathrm{N}_{7}$ & $\begin{array}{c}1.388 \pm \\
0.006\end{array}$ & 1.384 & 1.382 & 1.384 & 1.385 & 1.384 & 1.386 \\
\hline $\mathrm{N}_{7}-\mathrm{C}_{8}$ & $\begin{array}{c}1.305 \pm \\
0.006\end{array}$ & 1.308 & 1.309 & 1.308 & 1.308 & 1.307 & 1.309 \\
\hline $\mathrm{C}_{8}-\mathrm{N}_{9}$ & $\begin{array}{c}1.374 \pm \\
0.007\end{array}$ & 1.376 & 1.377 & 1.376 & 1.376 & 1.376 & 1.373 \\
\hline $\mathrm{N}_{9}-\mathrm{C}_{4}$ & $\begin{array}{c}1.375 \pm \\
0.008\end{array}$ & & & & & & \\
\hline $\mathrm{C}_{8}-\mathrm{H}_{8}$ & - & 1.079 & 1.079 & 1.079 & 1.079 & 1.079 & 1.079 \\
\hline $\mathrm{N}_{1}-\mathrm{H}_{1}$ & - & 1.013 & 1.012 & 1.030 & 1.026 & 1.025 & 1.025 \\
\hline $\mathrm{N}_{2}-\mathrm{H}_{2 \mathrm{a}}$ & - & 1.009 & 1.009 & 1.009 & 1.021 & 1.020 & 1.020 \\
\hline $\mathrm{N}_{2}-\mathrm{H}_{2 b}$ & - & 1.009 & 1.009 & 1.014 & 1.014 & 1.019 & 1.019 \\
\hline $\mathrm{N}_{9}-\mathrm{C}_{\mathrm{me}}$ & $\begin{array}{c}1.459 \pm \\
0.009 \\
\end{array}$ & - & 1.456 & 1.457 & 1.457 & 1.458 & - \\
\hline $\mathrm{N}_{9}-\mathrm{H}_{9}$ & - & 1.010 & - & - & - & - & 1.029 \\
\hline
\end{tabular}

${ }^{1}$ Bond distances (in $\AA$ ) averaged over 21 high resolution crystal structures of different Gua chromophores, retrieved form Nucleic Acid Database, and described in Ref $72^{1} ; \mathrm{C}_{1}{ }^{\prime}$ and $\mathrm{C}_{\mathrm{me}}$ stand for carbon atom of glycosidic bond in guanine nucleoside and that of methyl group in 9meG. Bonds that undergo maximum changes due to inclusion of explicit water are labeled in bold font. 
Table S9. Experimental and computed vibrational wavenumbers of GMP. Vibrational frequencies are computed on $\mathrm{N}_{9} \mathrm{H}-\mathrm{Gua}$, $\mathrm{N}_{9} \mathrm{H}-\mathrm{Gua} \cdot 7 \mathrm{H}_{2} \mathrm{O}$ and $9-\mathrm{meG} \cdot 6 \mathrm{H}_{2} \mathrm{O}$ model complex with B3LYP density functional.

\begin{tabular}{|c|c|c|c|c|c|c|c|c|}
\hline \multicolumn{3}{|c|}{ Experiment } & \multicolumn{3}{|c|}{$\mathrm{N}_{9} \mathrm{H}-\mathrm{Gua}$} & \multirow{2}{*}{$\frac{\mathrm{N}_{9} \mathrm{H}-\mathrm{Gua} \cdot 7 \mathrm{H}_{2} \mathrm{O}}{\begin{array}{c}\text { Freq } \\
\left(\mathrm{cm}^{-1}\right)\end{array}}$} & \multirow{2}{*}{$\begin{array}{c}\text { 9-meG } \\
\text { Freq } \\
\left(\mathrm{cm}^{-1}\right)\end{array}$} & \multirow{2}{*}{ 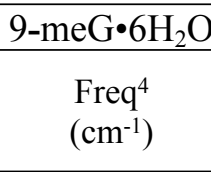 } \\
\hline $\begin{array}{c}\text { GMP, } \\
\text { RR, } \\
260 \mathrm{~nm}^{1}\end{array}$ & $\begin{array}{c}\text { GMP, } \\
\text { RR, } \\
210 \mathrm{~nm}^{1}\end{array}$ & $\begin{array}{c}\text { Gua, } \\
\text { NRS, } \\
632.8 \mathrm{~nm}^{2}\end{array}$ & $\begin{array}{l}\text { Freq }^{3} \\
\left(\mathrm{~cm}^{-1}\right)\end{array}$ & $\begin{array}{l}\text { Freq }^{4} \\
\left(\mathrm{~cm}^{-1}\right)\end{array}$ & $\begin{array}{l}\text { Freq } \\
\left(\mathrm{cm}^{-1}\right)\end{array}$ & & & \\
\hline 1681 & 1689 & 1675 & 1831 & 1697 & 1797 & 1665 & 1696 & 1665 \\
\hline 1602 & 1607 & 1602 & 1674 & 1641 & 1667 & 1702 & 1643 & 1703 \\
\hline 1576 & 1580 & 1551 & 1623 & 1592 & 1621 & 1623 & 1598 & 1622 \\
\hline 1537 & 1539 & 1479 & 1567 & 1550 & 1559 & 1554 & 1563 & 1566 \\
\hline 1486 & 1485 & 1468 & 1524 & 1500 & 1517 & 1499 & 1499 & 1499 \\
\hline 1412 & 1414 & 1421 & 1448 & 1421 & 1441 & 1422 & 1425 & 1428 \\
\hline 1366 & 1365 & 1361 & 1368 & 1356 & 1365 & 1357 & 1383 & 1377 \\
\hline 1319 & 1319 & 1234 & 1312 & 1306 & 1310 & 1313 & 1302 & 1305 \\
\hline 1204 & 1209 & 1186 & 1178 & 1177 & 1176 & 1202 & 1157 & 1189 \\
\hline 1204 & 1209 & - & - & - & - & - & 1247 & 1251 \\
\hline 1173 & 1176 & 1159 & 1146 & 1147 & 1141 & 1174 & 1112 & 1132 \\
\hline 1173 & 1176 & - & - & 1147 & 1176 & 1202 & 1157 & 1189 \\
\hline 1111 & 1121 & 1048 & 1075 & 1084 & 1074 & 1137 & - & 1068 \\
\hline 1079 & 1078 & 1048 & 1077 & 1093 & 1076 & 1130 & - & 1132 \\
\hline 1015 & 1034 & 1048 & 1047 & 1043 & 1047 & 1065 & 1074 & 1088 \\
\hline 1020 & - & 937 & 946 & 951 & 945 & 962 & 1026 & 1035 \\
\hline 868 & 866 & - & 836 & 835 & 835 & 852 & 856 & 871 \\
\hline- & 822 & - & 817 & 851 & 822 & 862 & 848 & 855 \\
\hline 789 & 794 & - & 773 & 798 & 765 & 800 & 797 & 799 \\
\hline
\end{tabular}

${ }^{1}$ from Ref 96; ${ }^{2}$ Normal Raman spectrum (NRS) of polycrystalline guanine, from Ref 47; ${ }^{3}$ computed at B3LYP/6-31G(d,p) level and not scaled, from Ref. $61 ;{ }^{4}$ current study, computed at B3LYP/6-311+G(2d,p)//PCM level and frequencies are not scaled; 5 computed at B3LYP/6$311++\mathrm{G}(\mathrm{d}, \mathrm{p}) / /$ vacuo level and frequencies are not scaled, from Ref. 47. 
Table S10. Computed vertical singlet excitation energies $(\Delta \mathrm{E}$ in $\mathrm{nm}$ and $\mathrm{eV})$, oscillator strengths $\left(\mathrm{f}_{\mathrm{PCM}}\right)$ and major orbital contribution $^{1}$ of key electronic transitions of GMP computed on 9-meG $\mathbf{9} \mathbf{6} \mathbf{H}_{\mathbf{2}} \mathbf{O}$ model by LR-TD-DFT using three different functional with 6-311+G(2d,p) basis set and implicit PCM solvation.

\begin{tabular}{|c|c|c|c|c|c|c|c|c|c|c|c|c|}
\hline & \multicolumn{3}{|c|}{ PBE0 } & \multicolumn{3}{|c|}{ CAM-B3LYP } & \multicolumn{3}{|c|}{$\omega \mathrm{B} 97 \mathrm{xD}$} & \multicolumn{2}{|c|}{$\mathrm{CASSCF} / \mathrm{CASPT} 2^{2}$} & \multirow{2}{*}{$\begin{array}{c}\operatorname{Exp}^{3} \\
\Delta \mathrm{E}_{\operatorname{Exp}} \\
\mathrm{eV}\end{array}$} \\
\hline Type & $\begin{array}{l}\text { State } \\
\text { order }\end{array}$ & $\begin{array}{c}\text { Major orbital } \\
\text { Contribution } \\
{ }^{1}(\%)\end{array}$ & $\begin{array}{c}\Delta \mathrm{E}_{\mathrm{vert}, \mathrm{PCM}} \\
\mathrm{eV} \\
\left(\mathrm{f}_{\mathrm{PCM}}\right)\end{array}$ & $\begin{array}{l}\text { State } \\
\text { order }\end{array}$ & $\begin{array}{c}\text { Major orbital } \\
\text { Contribution }{ }^{1} \\
(\%)\end{array}$ & $\begin{array}{c}\Delta \mathrm{E}_{\text {vert,PCM }} \\
\mathrm{eV} \\
\left(\mathrm{f}_{\mathrm{PCM}}\right)\end{array}$ & $\begin{array}{l}\text { State } \\
\text { order }\end{array}$ & $\begin{array}{l}\text { Major orbital } \\
\text { Contribution } \\
\quad(\%)\end{array}$ & $\begin{array}{c}\Delta \mathrm{E}_{\text {vert,PCM }} \\
\mathrm{eV} \\
\left(\mathrm{f}_{\mathrm{PCM}}\right)\end{array}$ & $\Delta \mathrm{E}(\mathrm{f})$ & $\begin{array}{c}\text { CSF } \\
\text { (weights) }\end{array}$ & \\
\hline $\begin{array}{c}L_{a} \\
\left(\pi \pi^{*}\right)\end{array}$ & S1 & $\mathrm{H} \rightarrow \mathrm{L}(92)$ & $\begin{array}{c}4.69 \\
(0.16) \\
\end{array}$ & S1 & $\mathrm{H} \rightarrow \mathrm{L}(85)$ & $\begin{array}{c}4.86 \\
(0.19) \\
\end{array}$ & S1 & $\mathrm{H} \rightarrow \mathrm{L}(86)$ & $\begin{array}{c}4.87 \\
(0.18)\end{array}$ & $\begin{array}{c}4.73 \\
(0.15) \\
\end{array}$ & $\mathrm{H} \rightarrow \mathrm{L}(68)$ & 4.47 \\
\hline $\begin{array}{c}L_{b} \\
\left(\pi \pi^{*}\right)\end{array}$ & S2 & $\mathrm{H} \rightarrow \mathrm{L}+1(90)$ & $\begin{array}{c}5.21 \\
(0.34)\end{array}$ & S2 & $\mathrm{H} \rightarrow \mathrm{L}+2(82)^{4}$ & $\begin{array}{c}5.4 \\
(0.42)\end{array}$ & S2 & $\mathrm{H} \rightarrow \mathrm{L}+1(83)$ & $\begin{array}{c}5.44 \\
(0.42)\end{array}$ & $\begin{array}{c}5.11 \\
(0.24)\end{array}$ & $\mathrm{H} \rightarrow \mathrm{L}+1(69)$ & 5.00 \\
\hline${ }^{1} \mathrm{n} \pi^{*}$ & S4 & $\mathrm{H}-2 \rightarrow \mathrm{L}(75)$ & $\begin{array}{c}5.74 \\
(0.002)\end{array}$ & S4 & $\begin{array}{l}\mathrm{H}-2 \rightarrow \mathrm{L}(56) \\
\mathrm{H}-4 \rightarrow \mathrm{L}(32)\end{array}$ & $\begin{array}{c}5.97 \\
(0.002)\end{array}$ & S3 & $\begin{array}{l}\mathrm{H}-2 \rightarrow \mathrm{L}(57) \\
\mathrm{H}-4 \rightarrow \mathrm{L}(22)\end{array}$ & $\begin{array}{c}5.97 \\
(0.0001)\end{array}$ & $\begin{array}{c}5.79 \\
(0.0001)\end{array}$ & - & 5.58 \\
\hline $\begin{array}{c}B_{b} \\
\left(\pi \pi^{*}\right)\end{array}$ & S9 & $\mathrm{H}-1 \rightarrow \mathrm{L}(80)$ & $\begin{array}{c}6.23 \\
(0.33)\end{array}$ & S8 & $\begin{array}{c}\mathrm{H}-1 \rightarrow \mathrm{L}(67) \\
\mathrm{H} \rightarrow \mathrm{L}+5 \text { (9) }\end{array}$ & $\begin{array}{c}6.54 \\
(0.29)\end{array}$ & S7 & $\begin{array}{c}\mathrm{H}-1 \rightarrow \mathrm{L}(49) \\
\mathrm{H} \rightarrow \mathrm{L}+5(32)\end{array}$ & $\begin{array}{c}6.58 \\
(0.22)\end{array}$ & $\begin{array}{c}6.49 \\
(0.29)\end{array}$ & $\begin{array}{c}\mathrm{H}-1 \rightarrow \mathrm{L}(51) \\
\mathrm{H}-1 \rightarrow \mathrm{L}+1(14)\end{array}$ & 6.21 \\
\hline
\end{tabular}

${ }^{1}$ Percentages contributions are calculated as $100 \mathrm{x}$ twice the squares of the coefficients in the CI expansion of TDDFT wave functions; ${ }^{2}$ Ref. 85, using CASSCF//CASPT2; ${ }^{3}$ Average experimental transition energies are derived from absorption in liquid, linear dichroism, circular dichroism, magnetic circular dichroism, and polarized absorption spectroscopy experiments, see Ref. 85 for details; ${ }^{4}$ with CAM-B3LYP functional $\mathrm{L}+1$ orbital (in B3LYP, PBE0, wB97xD) is reordered as L+2; Abbreviations; CSF, configuration state functions at CASASCF/CASPT2 level, LR, linear response; $\mathrm{H}$ and L; highest occupied molecular orbital (HOMO) and lowest unoccupied molecular orbital (LUMO) respectively. 
Table S11. Harmonic potential energy distribution (PED) of computed vibrational modes on three models of GMP; 9-meG, 9$\mathrm{meG} \bullet 4 \mathrm{H}_{2} \mathrm{O}$ and $9-\mathrm{meG} \bullet 6 \mathrm{H}_{2} \mathrm{O}$. Vibrational frequencies and normal modes are computed at B3LYP/6-311+G(2d,p)//PCM level.

\begin{tabular}{|c|c|c|c|c|c|c|c|}
\hline \multicolumn{2}{|c|}{ GMP, RR } & \multicolumn{2}{|r|}{ 9-meG } & \multicolumn{2}{|r|}{$9-\mathrm{meG} \bullet 4 \mathrm{H}_{2} \mathrm{O}$} & \multicolumn{2}{|r|}{$9-\mathrm{meG} \cdot 6 \mathrm{H}_{2} \mathrm{O}$} \\
\hline $\begin{array}{c}\lambda_{\mathrm{exc}}= \\
260 \mathrm{~nm}^{1}\end{array}$ & $\begin{array}{c}\lambda_{\text {exc }}= \\
210 \\
\mathrm{~nm}^{1}\end{array}$ & $\begin{array}{l}\text { Freq } \\
\left(\mathrm{cm}^{-1}\right)\end{array}$ & PED (\%) & $\begin{array}{l}\text { Freq } \\
\left(\mathrm{cm}^{-1}\right)\end{array}$ & PED (\%) & $\begin{array}{l}\text { Freq } \\
\left(\mathrm{cm}^{-1}\right)\end{array}$ & PED (\%) \\
\hline 1681 & 1689 & 1696 & $\begin{array}{l}\text { str O6C6 (54) - be } \\
\text { C6N1C2 (16) + str N3C2 } \\
\text { (7) }\end{array}$ & 1673 & $\begin{array}{l}\text { - str O6C6 (11) + be H1N1C2 } \\
(15)\end{array}$ & 1665 & $\begin{array}{l}\text { str O6C6 (15) - } \\
\text { be H1N1C2 (15) - be C6N1C2 (8) }\end{array}$ \\
\hline 1602 & 1607 & 1643 & be $\mathrm{H} 2 \mathrm{aN} 2 \mathrm{H} 2 \mathrm{~b}(75)$ & 1658 & $\begin{array}{l}\text { str C2N2 (10) + be } \\
\text { H2aN2H2b (35) }\end{array}$ & 1703 & $\begin{array}{l}\mathrm{H} 2 \mathrm{aN} 2 \mathrm{H} 2 \mathrm{~b}(48)+ \\
\text { be N2H2aO (10) }\end{array}$ \\
\hline 1537 & 1539 & 1563 & $\begin{array}{l}\text { str N7C8 }(15)-\text { be } \\
\text { N3C4C5 }(12)+\text { be } \\
\text { C4N9C8 (16) }\end{array}$ & 1568 & $\begin{array}{l}\text { - str O6C6 (12)+ str N7C8 (16) } \\
\text { - be H8C8N7 (11) + be } \\
\text { C4N9C8 (15) }\end{array}$ & 1566 & $\begin{array}{l}\text { str N7C8 }(18)+ \\
\text { be C4N9C8 (18) + } \\
\text { be H8C8N9 (12) } \\
\end{array}$ \\
\hline 1486 & 1485 & 1499 & $\begin{array}{l}\text { str O6C6(4) + str N7C8 } \\
(9)+\text { be } \mathrm{HCmeH}(24)\end{array}$ & 1510 & $\begin{array}{l}\text { str O6C6 (14) + str N7C8 (11) } \\
+ \text { str } \mathrm{N} 3 \mathrm{C} 2(11)-\text { be } \mathrm{HC}_{\mathrm{me}} \mathrm{H} \\
(13)\end{array}$ & 1499 & $\begin{array}{l}\text { str O6C6(13)+ str N7C8(12)+ str } \\
\mathrm{N} 3 \mathrm{C} 2(9)+\text { be } \mathrm{HC}_{\mathrm{me}} \mathrm{H}(11)\end{array}$ \\
\hline 1412 & 1414 & 1425 & $\begin{array}{l}\text { str N9C4 }(24)+\text { str N1C2 } \\
(9)-\operatorname{str} \mathrm{N}_{\mathrm{me}}(8)-\mathrm{be} \\
\text { C5N7C8 (13) }\end{array}$ & 1433 & $\begin{array}{l}- \text { str N9C4 (23) + be C5N7C8 } \\
(14)+\text { be C4N9C8 (8) - str } \\
\text { N1C2 (6) + str N9C } \text { me }_{\text {me }}(6)+\text { be } \\
\text { H1N1C2 (5) }\end{array}$ & 1428 & $\begin{array}{l}\text { str N9C4 (21) - be H1N1C2 (10) - be } \\
\text { C }_{m e} \text { N9C8 (10) - str N9C } C_{m e}(8)\end{array}$ \\
\hline 1366 & 1365 & 1383 & $\begin{array}{l}\text { str N9C8 (10) + be } \\
\text { C5N7C8 (14) }\end{array}$ & 1381 & $\begin{array}{l}\text { str N9C8 (12) + be C5N7C8 } \\
(13)\end{array}$ & 1377 & str N9C8(12) + be C5N7C8(16) \\
\hline 1319 & 1319 & 1302 & $\begin{array}{l}- \text { str N7C8 (15) - str } \\
\text { N3C4 (11) + str N7C5 } \\
(15) \text { - be H8C8N7 (19) }\end{array}$ & 1308 & $\begin{array}{l}\text { - str N7C8 (14) - str N3C4 (10) } \\
+ \text { str N7C5 (18) - be H8C8N7 } \\
(21)\end{array}$ & 1305 & $\begin{array}{l}-\operatorname{str} \mathrm{N} 7 \mathrm{C} 8(13)-\operatorname{str} \mathrm{N} 3 \mathrm{C} 4(12)+\mathrm{str} \\
\mathrm{N} 7 \mathrm{C} 5(19)+\text { be H8C8N7 (18) }\end{array}$ \\
\hline 1204 & 1209 & 1157 & $\begin{array}{l}- \text { str N7C5 (13) + str } \\
\text { N1C6 (33) - be C6N1C2 } \\
(10)\end{array}$ & 1192 & $\begin{array}{l}\text { - str N7C5 (15)+ str N1C6 (45) } \\
\text { - be C6N1C2 (5) }\end{array}$ & 1189 & $\begin{array}{l}-\operatorname{str} \text { N7C5 (14) + str N1C6 (46) - be } \\
\text { C6N1C2 (8) }\end{array}$ \\
\hline 1204 & 1209 & 1247 & $\begin{array}{l}\text { str }{\mathrm{N} 9 C_{\mathrm{me}}}(16)+\text { be } \\
\mathrm{H} 8 \mathrm{C} 8 \mathrm{~N} 7(41)-\mathrm{be} \\
\mathrm{N} 7 \mathrm{C} 8 \mathrm{~N} 9(14)\end{array}$ & 1253 & $\begin{array}{l}\text { str } \mathrm{N} \mathrm{C}_{\mathrm{me}}(16)+\text { be H8C8N7 } \\
(35) \text { - be N7C8N9 (15) }\end{array}$ & 1251 & $\begin{array}{l}\text { - str N9Cme (18) + be H8C8N9 (29) + } \\
\text { be C5N7C8 (20) }\end{array}$ \\
\hline 1079 & 1078 & - & & 1130 & $\begin{array}{l}\text { - str N3C2 (11) + be H2bN2C2 } \\
(43) \text { - be H2aN2O explicit }(11)- \\
\text { str C2N2 (5) + str N1C2 (5) }\end{array}$ & 1132 & $\begin{array}{l}- \text { str N3C2 (20) + str N1C2 (10) + be } \\
\text { H2bN2O }\end{array}$ \\
\hline 1015 & 1034 & 1074 & $\begin{array}{l}\text { str N1C2(12) - str } \\
\text { N1C6(19) + be } \\
\text { C5N7C8(11) - be } \\
\text { C2N3C4(12) }\end{array}$ & 1098 & $\begin{array}{l}\text { - str N1C2 (13) + be C2N3C4 } \\
(14) \text { - be C5N7C8 (10) }\end{array}$ & 1088 & $\begin{array}{l}\text { - str N1C2 (11)+ be C2N3C4 (15) - } \\
\text { be C5N7C8 (12) }\end{array}$ \\
\hline
\end{tabular}




\begin{tabular}{|c|c|c|c|c|c|c|c|}
\hline 1020 & - & 1026 & $\begin{array}{l}- \text { str N9C4 }(10)+\text { str } \\
\text { N1C2 }(24)-\text { be C5N7C8 } \\
(11)-\text { be H2bN2C2 (10) }\end{array}$ & 1042 & $\begin{array}{c}\text { str N1C2 (14) - str N9C4 (11)+ } \\
\text { str N2C2 (7) + str N3C2 (9) - } \\
\text { be C5N7C8 (13) }\end{array}$ & 1035 & $\begin{array}{l}\text { str N1C2 }(21)+\operatorname{str} \mathrm{N} 3 \mathrm{C} 2(11)-\mathrm{str} \\
\text { N9C4 (13) + str N2C2 (8) - be } \\
\text { C5N7C8 (11) }\end{array}$ \\
\hline 868 & 866 & 856 & $\begin{array}{l}\text { str N9C8 }(11)+\text { str N3C4 } \\
(11)+\text { be N3C4C5 }(12)+ \\
\text { be N1C2N3 (10)+ be } \\
\text { N9C8N7 (12) }\end{array}$ & 869 & $\begin{array}{l}\text { - str N9C8 (12) - str N3C4 (9) - } \\
\text { be N7C8N9 (12) - be N3C4C5 } \\
(10) \text { - be N1C2C3 (9) - be } \\
\text { C4N9C8 (8) }\end{array}$ & 871 & $\begin{array}{l}\text { str N9C8 }(10)+\text { str N3C4 }(8)+\text { be } \\
\text { N7C8N9 }(10)+\text { be C5N7C8 (11) + be } \\
\text { C4N9C8 }(7)+\text { be N3C4C5 (8) - be } \\
\text { C2N3C4 } 5) \text { - be C5N7C8 (5) }\end{array}$ \\
\hline 789 & 794 & 797 & $\begin{array}{l}\text { tors N1C2N3C4 (8) - tors } \\
\text { C5N7C8N9 (23) - out } \\
\text { O6N1C5C6 (35) - out } \\
\text { N3N9C5C4 (21) }\end{array}$ & 801 & $\begin{array}{l}\text { - tors C5N7C8N9 (20) - out } \\
\text { O6N1C5C6 (33) - out } \\
\text { N3N9C5C4 (25) }\end{array}$ & 799 & $\begin{array}{l}\text { tors N1C2N3C4 }(16)+\text { tors } \\
\text { C5N7C8N9 (17) + out N3N9C5C4 } \\
(42)\end{array}$ \\
\hline
\end{tabular}

Abbreviations; str, stretching mode; be, bending mode, tors, torsional mode; out, out of plane mode; $\mathrm{O}_{\text {explicit }}$, oxygen atom of explicit water molecule, $\mathrm{C}_{\mathrm{me}}$, carbon atom of the methyl group at $\mathrm{N} 9$; the signs before internal coordinate contributions represents relative direction with respect to other coordinates in the same PED. 
Table S12. Computed vertical singlet excitation energies $(\Delta \mathrm{E}$ in $\mathrm{nm}$ and $\mathrm{eV})$, oscillator strengths $\left(\mathrm{f}_{\mathrm{PCM}}\right)$ and major orbital contribution $^{1}$ of key $\pi \pi^{*}$ electronic transitions of GMP computed on N9H-Gua $\cdot 7 \mathrm{H}_{2} \mathrm{O}$ model by TD-B3LYP method on ground state geometry obtained with B3LYP functional, and 6-31G(d,p), 6-311+G(2d,p) and aug-ccpVTZ basis sets, with PCM implicit solvation.

\begin{tabular}{|c|c|c|c|c|c|c|c|c|c|c|c|c|}
\hline & \multicolumn{9}{|c|}{ Excited State with TD-B3LYP on N9H-Gua $\cdot 7 \mathrm{H}_{2} \mathrm{O}$} & \multirow{2}{*}{\multicolumn{2}{|c|}{$\begin{array}{l}\text { Excited State with } \\
\text { CASSCF/CASPT2 }^{2}\end{array}$}} & \multirow{3}{*}{$\begin{array}{c}\operatorname{Exp}^{3} \\
\Delta \mathrm{E}_{\text {Exp }} \\
\mathrm{eV}\end{array}$} \\
\hline & \multicolumn{3}{|c|}{$6-311+\mathrm{G}(2 \mathrm{~d}, \mathrm{p})$} & \multicolumn{3}{|c|}{$6-31 \mathrm{G}(\mathrm{d}, \mathrm{p})$} & \multicolumn{3}{|c|}{ aug-cc-pVTZ } & & & \\
\hline Type & $\begin{array}{l}\text { State } \\
\text { order }\end{array}$ & $\begin{array}{l}\text { Major orbital } \\
\text { Contribution } \\
\quad 1(\%)\end{array}$ & $\begin{array}{c}\Delta \mathrm{E}_{\text {vert,PCM }} \\
\mathrm{eV} \\
\left(\mathrm{f}_{\mathrm{PCM}}\right)\end{array}$ & $\begin{array}{l}\text { State } \\
\text { order }\end{array}$ & $\begin{array}{l}\text { Major orbital } \\
\text { Contribution } \\
\quad(\%)\end{array}$ & $\begin{array}{c}\Delta \mathrm{E}_{\text {vert,PCM }} \\
e V \\
\left(f_{\mathrm{PCM}}\right)\end{array}$ & $\begin{array}{l}\text { State } \\
\text { order }\end{array}$ & $\begin{array}{l}\text { Major orbital } \\
\text { Contribution } \\
\quad(\%)\end{array}$ & $\begin{array}{c}\Delta \mathrm{E}_{\text {vert, } \mathrm{PCM}} \\
\mathrm{eV} \\
\left(\mathrm{f}_{\mathrm{PCM}}\right)\end{array}$ & $\Delta \mathrm{E}(\mathrm{f})$ & $\begin{array}{c}\text { CSF } \\
\text { (weights) }\end{array}$ & \\
\hline $\begin{array}{c}L_{a} \\
\left(\pi \pi^{*}\right)\end{array}$ & S1 & $\mathrm{H} \rightarrow \mathrm{L}(93)$ & $\begin{array}{c}4.59 \\
(0.15)\end{array}$ & S1 & $\mathrm{H} \rightarrow \mathrm{L}(93)$ & $\begin{array}{c}4.54 \\
(0.16)\end{array}$ & $\mathrm{S} 1$ & $\mathrm{H} \rightarrow \mathrm{L}(93)$ & $\begin{array}{c}4.59 \\
(0.16)\end{array}$ & $\begin{array}{c}4.73 \\
(0.15)\end{array}$ & $\mathrm{H} \rightarrow \mathrm{L}(68)$ & 4.47 \\
\hline $\begin{array}{c}L_{b} \\
\left(\pi \pi^{*}\right)\end{array}$ & S2 & $\mathrm{H} \rightarrow \mathrm{L}+1(91)$ & $\begin{array}{c}5.17 \\
(0.34)\end{array}$ & S2 & $\mathrm{H} \rightarrow \mathrm{L}+1$ (49) & $\begin{array}{c}5.4 \\
(0.42)\end{array}$ & S2 & $\mathrm{H} \rightarrow \mathrm{L}+1(91)$ & $\begin{array}{c}5.18 \\
(0.34)\end{array}$ & $\begin{array}{c}5.11 \\
(0.24)\end{array}$ & $\mathrm{H} \rightarrow \mathrm{L}+1$ (69) & 5.00 \\
\hline${ }^{1} \mathrm{n} \pi^{*}$ & S5 & $\mathrm{H}-1 \rightarrow \mathrm{L}(73)$ & $\begin{array}{c}5.64 \\
(0.0004)\end{array}$ & S5 & $\mathrm{H}-1 \rightarrow \mathrm{L}(79)$ & $\begin{array}{c}5.63 \\
(0.0002)\end{array}$ & S5 & $\mathrm{H}-1 \rightarrow \mathrm{L}(77)$ & $\begin{array}{c}5.65 \\
(0.0003)\end{array}$ & $\begin{array}{c}5.79 \\
(0.0001)\end{array}$ & - & 5.58 \\
\hline $\begin{array}{c}B_{b} \\
\left(\pi \pi^{*}\right)\end{array}$ & $\mathrm{S} 10$ & $\begin{array}{l}\mathrm{H}-2 \rightarrow \mathrm{L}(54) \\
\mathrm{H}-3 \rightarrow \mathrm{L}(20)\end{array}$ & $\begin{array}{c}6.26 \\
(0.25)\end{array}$ & S8 & $\begin{aligned} \mathrm{H}-2 & \rightarrow \mathrm{L}(45) \\
\mathrm{H}-3 & \rightarrow \mathrm{L}(26)\end{aligned}$ & $\begin{array}{c}6.22 \\
(0.29)\end{array}$ & S10 & $\begin{array}{l}\mathrm{H}-2 \rightarrow \mathrm{L}(57) \\
\mathrm{H}-3 \rightarrow \mathrm{L}(20)\end{array}$ & $\begin{array}{c}6.27 \\
(0.26)\end{array}$ & $\begin{array}{c}6.49 \\
(0.29)\end{array}$ & $\begin{array}{c}\mathrm{H}-1 \rightarrow \mathrm{L}(51) \\
\mathrm{H}-1 \rightarrow \mathrm{L}+1(14)\end{array}$ & 6.21 \\
\hline
\end{tabular}

${ }^{1}$ Percentages contributions are calculated as $100 \mathrm{x}$ twice the squares of the coefficients in the CI expansion of TDDFT wave functions; ${ }^{2}$ Ref. 85, using CASSCF//CASPT2; ${ }^{3}$ Average experimental transition energies are derived from absorption in liquid, linear dichroism, circular dichroism, magnetic circular dichroism, and polarized absorption spectroscopy experiments, see Ref. 85 for details.

Abbreviations; CSF, configuration state functions at CASASCF/CASPT2 level, LR, linear response; H and L; highest occupied molecular orbital (HOMO) and lowest unoccupied molecular orbital (LUMO) respectively. 